\title{
Observer-based control of a class of chaotic systems
}

\author{
Ercan Solak, Ömer Morgül *, Umut Ersoy \\ Department of Electrical and Electronics Engineering, Bilkent University, 06533 Bilkent, Ankara, Turkey
}

Received 6 September 2000; accepted 5 December 2000

Communicated by A.P. Fordy

\begin{abstract}
We consider the control of a class of chaotic systems, which covers the forced chaotic oscillators. We focus on two control problems. The first one is to change the dynamics of the system to a new one which exhibits a desired behavior, and the second one is the tracking problem, i.e., to force the solutions of the chaotic system to track a given trajectory. To solve these problems we use observers which could be used to estimate the unknown states of the system to be controlled. We apply the proposed method to the control of Duffing equation and the Van der Pol oscillator and present some simulation results. @ 2001 Elsevier Science B.V. All rights reserved.
\end{abstract}

PACS: 05.45.+b

Keywords: Chaotic systems; Chaos synchronization; State observers; Feedback systems; Stabilization; Tracking

\section{Introduction}

Many different aspects of chaotic dynamics have attracted extensive interest from different disciplines in recent years. An interesting and challenging problem in the field is the control of chaotic systems. However, there is neither a general method, nor a common framework for this control problem. Many publications on the subject [1-3] focus on driving a system from a chaotic regime to periodic orbits and from periodic orbits to chaotic trajectories. Main control strategies that have been studied are open-loop methods [4], OGY technique [2,5] and control engineering tools $[1,6]$. There are yet other approaches that are more complex and difficult to apply in many cases $[7,8]$. An

\footnotetext{
* Corresponding author. Fax: 90-312-266-41-92.

E-mail addresses: ercan@ee.bilkent.edu.tr (E. Solak), morgul@ee.bilkent.edu.tr (Ö. Morgül).
}

extensive list of references on the subject can be found in [9].

In this Letter we present a state estimation and feedback approach to the control of a class of chaotic systems. The class we consider includes forced oscillators such as Duffing equation and the Van der Pol oscillator. We first transform these systems into a canonical form called the Brunowsky form, hence the method presented in this Letter may also be applied to systems which can be transformed into the Brunowsky form after a change of coordinates. Our aim is to alter the dynamics of the given chaotic system appropriately by using the control input to obtain a desirable behavior, i.e., to drive the system from chaos to periodic behavior, or vice versa. If the behavior of the system to be controlled depends on certain parameters in the dynamics, and if the bifurcation structure with respect to these parameters is known, then by applying input term appropriately, these coefficients can be adjusted to obtain a desirable behavior. Another prob- 
lem we address is forcing a chaotic system to track a reference trajectory. For both of these problems (control and tracking) we use state observers. Observers are dynamical systems which can be used to estimate the states of the system to be controlled. Such structures have also been used in the synchronization of chaotic systems, [10,11]. Robustness properties of observers used in chaotic synchronization were investigated in [12].

Then those estimates provided by the observer are used in state feedback to change the dynamics of the controlled system. This would bring an extra error term in the dynamics of the system to be controlled, however this error term also decays exponentially to zero, hence is not expected to change the asymptotic behavior of the system to be controlled.

A similar control problem was considered also in [1] and [13]. In both of these works, only the Duffing equation was considered, and their control problem was not to change the dynamics, but to force the solutions of the Duffing equation to track a given reference trajectory. Only in [13] a state observer was used, but both the form of the observer and the technique used are quite different than the ones considered in this Letter.

The Letter is organized as follows. In the next section we introduce the concept and basics of the state observers considered in this work. In Section 3 we consider the observer based control of forced oscillators. In Section 4 we apply the proposed control method to the control of Duffing equation and the Van der Pol oscillator and give simulation results. Finally, we give some concluding remarks.

\section{Full order observer}

We consider nonlinear systems having the following form:

$\dot{u}=A u+g(u)+h(t), \quad y=C u$,

where $A \in \mathbf{R}^{n \times n}, C \in \mathbf{R}^{1 \times n}$ are constant matrices, $y \in \mathbf{R}$ is the measured output, $g: \mathbf{R}^{n} \rightarrow \mathbf{R}^{n}$ is a differentiable nonlinear function and $h: \mathbf{R} \rightarrow \mathbf{R}^{n}$ is a known forcing function (and/or input).

For definitions of observability, observers and their applications to chaotic systems we refer to $[10,12]$ and the references therein.
We further impose the following structure on the system equation:

$A=\left(\begin{array}{ccccc}0 & 1 & 0 & \ldots & 0 \\ 0 & 0 & 1 & \ldots & 0 \\ & & \vdots & & \\ 0 & 0 & 0 & \ldots & 1 \\ 0 & 0 & 0 & \ldots & 0\end{array}\right)$,

$g(u)=\left(\begin{array}{c}0 \\ \vdots \\ 0 \\ 1\end{array}\right) f(u), \quad C=\left(\begin{array}{llll}1 & 0 & \ldots & 0\end{array}\right)$,

where $f: \mathbf{R}^{n} \rightarrow \mathbf{R}$ is a differentiable function that satisfies the Lipschitz property:

$\left\|f\left(u_{1}\right)-f\left(u_{2}\right)\right\| \leqslant \gamma\left\|u_{1}-u_{2}\right\|, \quad \forall u_{1}, u_{2} \in \mathbf{R}^{n}$,

for some positive $\gamma$. Here, $\|v\|$ represents standard Euclidean norm in $\mathbf{R}^{p}$ for any positive integer $p$ if $v \in \mathbf{R}^{p}$ and the induced matrix norm if $v \in \mathbf{R}^{p \times p}$. We note that, since all norms are equivalent in $\mathbf{R}^{p}$, the norm used in (3) is arbitrary.

The form given by (2) is called the Brunowsky canonical form, and is frequently used in the control of nonlinear systems; see, e.g., $[14,15]$. We note that some chaotic systems are already in this form; see, e.g., $[16,17]$ or can be transformed into this form, e.g., Rössler system. In the sequel we will consider forced chaotic oscillators and show that these oscillators can be readily transformed into this form.

To estimate the state vector $u(t)$ of system (1) we use the following observer:

$\dot{\hat{u}}=A \hat{u}+g(\hat{u})+L(y-C \hat{u})+h(t)$,

where $L \in \mathbf{R}^{n}$ is a gain vector chosen so that the error $\varepsilon=u-\hat{u}$ between the original state vector and its estimate converges to zero exponentially. The fact that such a gain vector can always be found is proved in [15] and a procedure to obtain the gain vector $L$ can be found in $[10,12]$. An improvement on the gain selection scheme is provided in [18]. 
Thus we have that the estimate $\hat{u}(t)$ approaches to $u(t)$ exponentially fast, i.e., the following holds for some $M>0$ and $\alpha>0$ :

$\|u(t)-\hat{u}(t)\| \leqslant M e^{-\alpha t}, \quad \forall t>0$.

This also shows that the proposed observer scheme can be used for the synchronization of chaotic systems given by (1).

In some special cases, the nonlinearity given in (1) may have the following special form:

$g(u)=\hat{g}(y), \quad y=C u$,

where $C$ is as given in (1), and $\hat{g}: \mathbf{R} \rightarrow \mathbf{R}^{n}$ is an arbitrary function. In this special case, the nonlinearity $g$ can be exactly constructed in the observer since $y$ is available from measurements. Then instead of the observer given by (4), we can use the following:

$\dot{\hat{u}}=A \hat{u}+\hat{g}(y)+L(y-\hat{y})+h(t), \quad \hat{y}=C \hat{u}$.

Hence by using (1), (6) and (7) we obtain the following error dynamics:

$\dot{\varepsilon}=(A-L C) \varepsilon$.

Fact 1. Consider the system given by (1) where the nonlinearity $g$ is of the form given by (6) and consider the observer given by (7). Assume that the pair $(C, A)$ is observable. Then there exists a feedback gain vector $L$ such that the error $\varepsilon$ decays globally exponentially to zero.

Proof. Choose a gain vector $L$ such that $A-L C$ is stable. Then the result follows from (8).

Remark 1. Note that Fact 1 holds for any nonlinearity $\hat{g}$, i.e., (3) need not be satisfied. Hence, $\hat{g}$ need not even be differentiable or continuous (e.g., can be a hysteresis or signum type nonlinearity). However, if we insist to use the observer given by (4), then obviously we need (3) to hold.

We note that some chaotic systems are already in this form, e.g., the systems in Lur'e form; see [19]. In the sequel we will show that the controlled Duffing equation can be transformed into this form.

\section{Observer-based control}

In this section we consider the application of the observer theory given in previous section to the control of a class of chaotic systems, namely forced chaotic oscillators. We note that this approach also applies to other class of systems, e.g., systems in Lur'e form, see $[16,19]$, or systems in Brunowsky canonical form, or any system which could be transformed into one of these forms. However, these classes will not be considered in this Letter.

We consider the systems given by the following equation:

$x^{(n)}+F\left(x, \dot{x}, \ldots, x^{(n-1)}\right)=h(t)+r(t)$,

where $x^{(i)}$ represents the $i$ th time derivative of $x$, $i=1,2, \ldots, n-1, h(t)$ is a known forcing function and $r(t)$ is the control input to be determined. We assume that $F$ is differentiable with respect to its arguments. This class of systems covers a wide range of chaotic oscillators, e.g., Duffing equation, Van der Pol equation, etc. Also, some class of systems (e.g., Lur'e class) can be reduced to this form. Introduction of the input term $r(t)$ in (9) is inspired by the works of [1] and [13], where the authors considered only the control of the Duffing equation.

We will first explain our methodology. Assume that the signal $x$ as well as its time derivatives $x^{(i)}, i=$ $1, \ldots, n-1$, are all available. Choose the control input $r$ as

$$
\begin{aligned}
r(t)= & \hat{h}(t)-h(t)+F\left(x, \ldots, x^{(n-1)}\right) \\
& -\hat{F}\left(x, \ldots, x^{(n-1)}\right),
\end{aligned}
$$

and substitute in (9) to get

$x^{(n)}+\hat{F}\left(x, \dot{x}, \ldots, x^{(n-1)}\right)=\hat{h}(t)$.

Hence we can convert the dynamics of the forced oscillator (9) to a desired dynamics given by (11) by the choice of the feedback input (10). This way, the dynamical behavior of (9) may be modified. A particular application of this idea is the following. Assume that $F$ in (9) has the form

$$
\begin{aligned}
F\left(x, \ldots, x^{(n-1)}\right)= & \sum_{i=1}^{p} \alpha_{i} F_{i}\left(x, \ldots, x^{(n-1)}\right) \\
& +F_{r}\left(x, \ldots, x^{(n-1)}\right),
\end{aligned}
$$


where, for $i=1,2, \ldots, p, F_{i}$ are known and differentiable functions, and $\alpha_{i}$ are real and constant parameters. Assume that the dynamical behavior of (9) depends on the parameters $\alpha_{i}$ (e.g., a bifurcation diagram in terms of parameters $\alpha_{i}$ is known). Then, by choosing the control input $r(t)$ as

$r(t)=\sum_{i}^{p}\left(\alpha_{i}-\hat{\alpha}_{i}\right) F_{i}+\hat{h}(t)-h(t)$,

(9) is reduced to

$x^{(n)}+\sum_{i}^{p} \hat{\alpha}_{i} F_{i}+F_{r}=\hat{h}(t)$,

hence any behavior in the bifurcation diagram of (9) can be obtained with appropriate choice of the parameters $\hat{\alpha}_{i}$.

The basic problem in the scheme presented above is the unavailability of the signals $x^{(i)}(t), i=1, \ldots$, $n-1$. We assume that $x(t)$ is measurable, however, obtaining the derivatives by numerical derivation is not desirable since inevitably this operation is adversely affected by the presence of noise in measurements. Alternatively we could use the observer theory given in the previous section to estimate the states, hence the derivatives. Moreover, the error between the estimates and the actual derivatives decay exponentially to zero. Hence in the control laws given by (10) or (13) we could use the estimates $\hat{x}^{(i)}, i=1, \ldots, n-1$. This would bring a perturbation term $\epsilon(t)$ in the right hand sides of (11) and (14), and assuming that the signals are bounded, this term $\epsilon(t)$ decays exponentially to zero. Hence, asymptotically we can neglect the term $\epsilon(t)$, and assuming that the behaviors of (11) and (14) (e.g., chaos, limit cycle, etc.) are structurally stable, we could expect to observe the similar behavior in the controlled system given by (11) or (14).

To elaborate further on structural stability, let us consider a perturbed version of (11),

$$
\begin{aligned}
x^{(n)} & +\hat{F}\left(x, \dot{x}, \ldots, x^{(n-1)}\right) \\
& =\hat{h}(t)+n\left(t, x, \dot{x}, \ldots, x^{n-1}\right),
\end{aligned}
$$

where $n: \mathbf{R}_{+} \times \mathbf{R}^{n} \rightarrow \mathbf{R}$ is an arbitrary smooth function. Let us assume that (11) exhibits certain behavior (e.g., chaos, limit cycle, etc.) in a bounded region $\Omega \subset$ $\mathbf{R}^{n}$. We say that this behavior is structurally stable if there exists $\epsilon_{0}>0$ such that, for any $n: \mathbf{R}_{+} \times \mathbf{R}^{n} \rightarrow \mathbf{R}$ satisfying $|n|<\epsilon_{0}$ on $\Omega$ and $\forall t>0$, solutions of (11) and (15) are topologically equivalent, i.e., a continuous and invertible function maps one to the other, see [20].

For formal derivations, we first transform (9) into the state space form by usual change of variables

$x_{1}=x, \quad x_{i+1}=x^{(i)}, \quad i=1,2, \ldots, n-1$.

Let $u=\left(\begin{array}{llll}x_{1} & x_{2} & \ldots & x_{n-1}\end{array}\right)^{\mathrm{T}}$ and define $f(u)=F\left(x_{1}\right.$, $\left.x_{2}, \ldots, x_{n-1}\right)$. With these definitions, state space representation of (9) is of the form (2). As before, we assume that $f$ satisfies the Lipschitz condition (3). In fact, since the systems considered in this Letter are chaotic, the solutions are bounded in a compact and convex region, and assuming the differentiability of $f$, in this region such a Lipschitz property holds. Hence, by using the Lipschitz constant $\gamma$ in (3) and results referred to in the previous section, a feedback gain $L$ can always be found so that (5) is satisfied. Defining $\hat{f}(\hat{u})=\hat{F}\left(\hat{x}_{1}, \hat{x}_{2}, \ldots, \hat{x}_{n-1}\right)$ we choose the control input $r$ as (cf. (10))

$r(t)=\hat{h}(t)-h(t)+f(\hat{u})-\hat{f}(\hat{u})$.

Although we do not have $u, \hat{u}$ is available, hence the control law (16) is implementable. Substituting this control law in (9) we obtain the expression of the controlled system as

$x^{(n)}+\hat{F}\left(x, \dot{x}, \ldots, x^{(n-1)}\right)=\hat{h}(t)+\epsilon(t)$,

where

$\epsilon(t)=[\hat{f}(u)-\hat{f}(\hat{u})]+[f(\hat{u})-f(u)]$.

Assuming that $\hat{f}$ is also Lipschitz, i.e., (3) is satisfied with a constant $\hat{\gamma}$, it follows from (3) and (5) that

$|\epsilon(t)| \leqslant(\gamma+\hat{\gamma}) M e^{-\alpha t}$.

Let $\epsilon_{0}>0$ be the bound on the perturbation term in (15) mentioned above. From (19) it follows easily that for $t>T=(1 / \alpha) \ln \left((\gamma+\hat{\gamma}) M / \epsilon_{0}\right)$ we have $\epsilon(t)<\epsilon_{0}$. Hence, we can apply $r(t)$ given by (16) for $t>T$, then by structural stability assumption the solutions of (11) and (17) will be topologically equivalent. Moreover, since $\epsilon(t)$ decays to zero exponentially fast, it is reasonable to expect the behaviors of (11) and (17) to be the same. 
In particular, assume that $F$ in (9) has the following special form:

$$
\begin{aligned}
& F\left(x, \dot{x}, \ldots, x^{(n-1)}\right) \\
& \quad=a_{0} x+a_{1} \dot{x}+\cdots+a_{n-1} x^{(n-1)}+f_{r}(x),
\end{aligned}
$$

where $a_{0}, \ldots, a_{n-1}$ are arbitrary real constants and $f_{r}$ is a differentiable function, not necessarily satisfying the Lipschitz property. After transforming to state space coordinates this system satisfies the conditions of Fact 1, hence instead of observer (4), we could use observer (7).

In [13] it was shown that by using a different observer structure, it is possible to design a controller so that the solutions of the Duffing equation tracks a given reference trajectory. The same approach can also be applied to any forced chaotic oscillator given by (9) by using the observers presented in this Letter.

Indeed, let $x_{d}(t)$ be a given reference trajectory which is sufficiently smooth. Let the chaotic forced oscillator be given by (9) and let $\hat{u}=\left(\hat{x}_{1} \hat{x}_{2} \ldots \hat{x}_{n-1}\right)^{\mathrm{T}}$ be the estimate of $u=\left(x_{1} x_{2} \ldots x_{n-1}\right)^{\mathrm{T}}$ provided by the observer. We choose the control law $r(t)$ as follows:

$$
\begin{aligned}
r(t)= & x_{d}^{n}(t)-k_{1}\left(\hat{x}-x_{d}\right)-k_{2}\left(\dot{\hat{x}}-\dot{x}_{d}\right)-\cdots \\
& -k_{n}\left(\hat{x}^{n-1}-x_{d}^{n-1}\right)-h(t)+F(\hat{u}) .
\end{aligned}
$$

We choose the tracking error $\varepsilon=x-x_{d}$ and using (21) in (9) and noting that $\hat{x}^{(i)}-x_{d}^{(i)}=\varepsilon^{(i)}+\hat{x}^{(i)}-x^{(i)}$, we obtain

$\varepsilon^{(n)}+k_{n} \varepsilon^{(n-1)}+\cdots+k_{1} \varepsilon=\delta(t)$,

where $\delta(t)$ is an exponentially decaying term, i.e., it satisfies $|\delta(t)| \leqslant M_{1} e^{-\alpha t}$ for some $M_{1}>0$, see (5). Let us choose the controller gains $k_{i}$ such that the roots of the polynomial $p(s)=s^{n}+k_{n} s^{n-1}+\cdots+k_{1}$ have all negative real parts. It easily follows that the solution of (22) decays exponentially to zero. Hence we will have $x^{(i)}(t) \rightarrow x_{d}^{(i)}(t), i=1,2, \ldots, n-1$. Moreover, the convergence is exponential.

In $[10,12]$, it was shown that the observer-based synchronization of chaotic systems is robust with respect to noise and parameter mismatch. In our methodology, in case of noise and/or parameter mismatch there will be an extra term in (17) and (22). This extra term is bounded and becomes smaller as the noise and/or parameter mismatch become smaller. Therefore its effect will be small provided that the noise and/or parameter mismatch is sufficiently small.

\section{Applications}

In this section, as an application of the ideas presented in the previous sections, we will consider the control of two well known forced chaotic oscillators, namely the forced Duffing equation and the Van der Pol oscillator.

\subsection{Duffing equation}

We consider the following system:

$\ddot{x}+a_{0} x+a_{1} \dot{x}+a_{2} x^{3}=q \cos \omega t+r(t)$.

For the uncontrolled case (i.e., $r=0$ ), the bifurcation structure of Duffing equation (23) with respect to parameters $a_{0}, a_{1}, a_{2}, q$ and $\omega$ can be found in many sources; see, e.g., [21]. The control of Duffing equation is considered in [13] and [1]. In [1], the proposed method for control is not based on an observer and the aim is not to change the dynamics of (23) (e.g., the parameters of the system), but to force the solutions of (23) to track a known solution $x_{d}(t), \dot{x}_{d}(t)$. In [13], the same problem is considered and a solution by using an observer is provided. Hence, the control problem considered here (e.g., to change the dynamics) is different than the control problem considered in these references (e.g., to track a reference trajectory). Moreover, the observer proposed in [13] is inspired by the same authors' work in robotics, and is quite different than the one considered in this Letter. Moreover, note that both in [13] and [1], only Duffing equation is considered, however our method applies to all forced chaotic oscillators in the form given by (9).

By comparing (9) and (23), it is obvious that the nonlinearity $F$ could be written in the form (20). Hence we can use either of the observers given by (4) or (7). We choose the latter, since in this case the error dynamics are linear.

Let us choose $x_{1}=x$ and $x_{2}=\dot{x}$. Then (23) can be rewritten as

$\dot{x}_{1}=x_{2}$,

$\dot{x}_{2}=-a_{0} x-a_{1} x_{2}-a_{2} x_{1}^{3}+q \cos \omega t+r(t)$, 
which is in the form given by (1) and (2) with $u=$ $\left(\begin{array}{ll}x_{1} & x_{2}\end{array}\right)^{\mathrm{T}}$ :

$A=\left(\begin{array}{cc}0 & 1 \\ -a_{0} & -a_{1}\end{array}\right), \quad f(y)=a_{2} y^{3}, \quad y=x_{1}$,

where $y=C u$ with $C=\left(\begin{array}{ll}1 & 0\end{array}\right)$. For the observer, we choose the form (7). Hence the observer is given by

$$
\begin{aligned}
\dot{\hat{x}}_{1}= & \hat{x}_{2}+l_{1}\left(x-\hat{x}_{1}\right), \\
\dot{\hat{x}}_{2}= & -a_{0} \hat{x}_{1}-a_{1} \hat{x}_{2}+l_{2}\left(x-\hat{x}_{1}\right)-a_{2} y^{3} \\
& +q \cos \omega t+r(t),
\end{aligned}
$$

where the gain vector $L=\left[\begin{array}{ll}l_{1} & l_{2}\end{array}\right]^{\mathrm{T}}$ should be chosen so that the matrix $A_{c}=A-L C$ is stable, see (8). Since the pair $(C, A)$ is observable, this is always possible. With this choice, the estimation errors $\mid x_{1}-$ $\hat{x}_{1} \mid$ and $\left|x_{2}-\hat{x}_{2}\right|$ decay exponentially to zero, see (8). Moreover, the decay rate can be adjusted arbitrarily by the choice of the gain vector $L$.

Now we can choose the input term $r(t)$ as (cf. (10) or (13))

$$
\begin{aligned}
r(t)= & -q \cos \omega t+\hat{q} \cos \hat{\omega} t+\left(a_{0}-\hat{a}_{0}\right) \hat{x}_{1} \\
& +\left(a_{1}-\hat{a}_{1}\right) \hat{x}_{2}+\left(a_{2}-\hat{a}_{2}\right) y^{3}
\end{aligned}
$$

and substitute in (23) to get the controlled dynamics as

$\ddot{x}+\hat{a}_{0} x+\hat{a}_{1} \dot{x}+\hat{a}_{2} x^{3}=\hat{q} \cos \hat{\omega} t+\epsilon(t)$,

where

$\epsilon(t)=\left(a_{0}-\hat{a}_{0}\right)\left(\hat{x}_{1}-x_{1}\right)+\left(a_{1}-\hat{a}_{1}\right)\left(\hat{x}_{2}-x_{2}\right)$.

Since we assume that $y=x$ is available from measurements, the control input could also be chosen as

$$
\begin{aligned}
r(t)= & -q \cos \omega t+\hat{q} \cos \hat{\omega} t+\left(a_{0}-\hat{a}_{0}\right) x \\
& +\left(a_{1}-\hat{a}_{1}\right) \hat{x}_{2}+\left(a_{2}-\hat{a}_{2}\right) y^{3} .
\end{aligned}
$$

Then (27) remains valid with $\epsilon(t)=\left(a_{1}-\hat{a}_{1}\right)\left(\hat{x}_{2}-\right.$ $\left.x_{2}\right)$.

For simulation we begin by setting the eigenvalues of $A_{c}$ as -1 and -2 . The $C$ matrix is ( 10$)$, therefore the corresponding vector becomes $L=\left[3-a_{1} 2-\right.$ $\left.3 a_{1}+a_{1}^{2}-a_{0}\right]^{\mathrm{T}}$. From the simulations we have seen that the states of the observer system given by Eqs. (24) and (25), converges to the states of the original system in about $10 \mathrm{~s}$. The convergence rate can be made larger by choosing the eigenvalues of $A_{c}$ further away from the imaginary axis. However, the eigenvalues given above yield acceptable performance since we deal with the steady state behavior of the system.

Then we select two sets of parameters of the Duffing system:

set $1=\left(a_{0}=0.25, a_{1}=0, a_{2}=1, q=11, \omega=1\right)$, set $2=\left(a_{0}=1.45, a_{1}=0, a_{2}=1, q=11, \omega=1\right)$.

From the bifurcation diagrams and formal analysis on the system $[1,21,22]$, the first set corresponds to chaotic regime and the latter to limit cycle. For the parameters set as in set 1, a typical system trajectory is shown in Fig. 1(a). We choose the control law as $r(t)=-1.2 \hat{x}_{2}$, where $\hat{x}$ denotes the states of the observer and is available. After the control, the original system exhibits the behavior corresponding to parameter set 2, which is shown in Fig. 1(b). The limit cycle figures are plotted for the time interval after the transients have died out.

\subsection{Forced Van der Pol oscillator}

As a second example, consider the following forced Van der Pol oscillator:

$\ddot{x}+d\left(x^{2}-1\right) \dot{x}+x=a \cos \omega t+r(t)$.

It was shown in [23] that for various values of $d, a$ and $\omega$, this oscillator exhibits a large variety of nonlinear phenomena, including chaos. This system is in the form given by (9) with

$F(x, \dot{x})=d\left(x^{2}-1\right) \dot{x}+x$.

We note that $F(x, \dot{x})$ in (29) is not a function of $x$ only, hence the observer given by (7) cannot be used. We first transform (28) to state space form by the usual coordinate change $x_{1}=x, x_{2}=\dot{x}$ and obtain

$\dot{x}_{1}=x_{2}$,

$\dot{x}_{2}=-d\left(x_{1}^{2}-1\right) x_{2}-x_{1}+a \cos \omega t+r(t)$.

The nonlinearity given by (29) is not globally Lipschitz. However, the solutions of (28) which are of interest to us are bounded in a convex region $B$, and by the differentiability of $F$ we may assume that (3) holds in $B$. Eqs. (30) and (31) can be put into the form (2) 


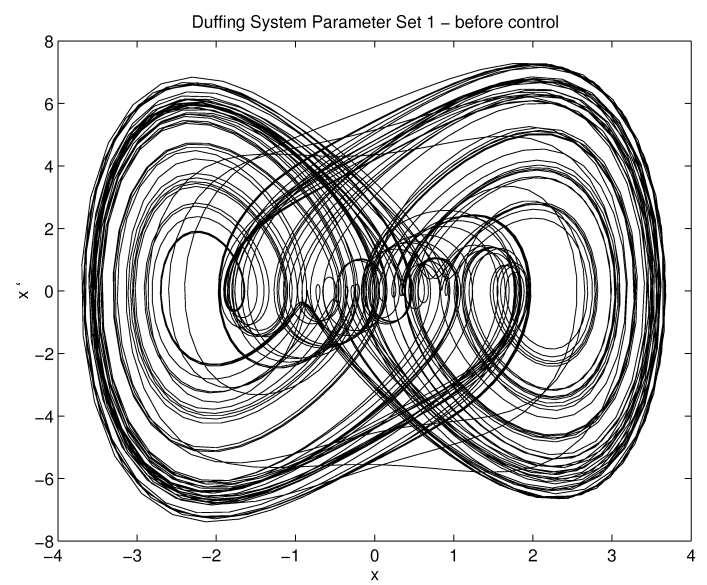

(a)

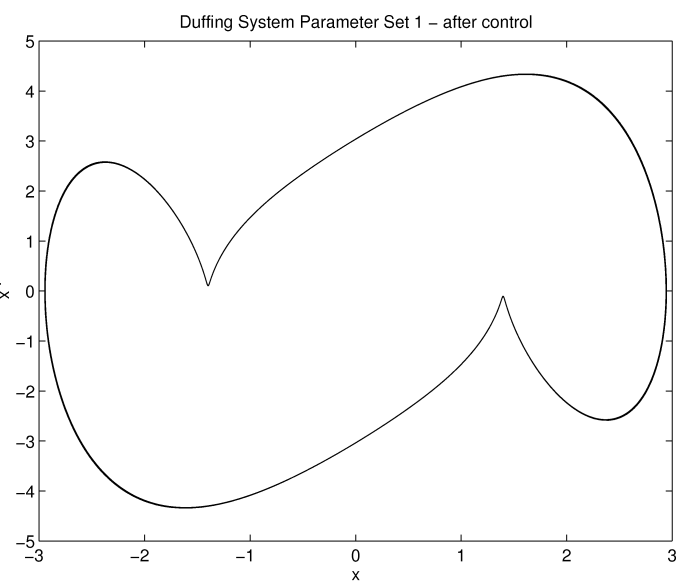

(b)

Fig. 1. State trajectories of the Duffing equation for set 1: (a) before control, (b) after control.

with $u=\left(x_{1} x_{2}\right)^{\mathrm{T}}$ and

$A=\left(\begin{array}{ll}0 & 1 \\ 0 & 0\end{array}\right), \quad f(y)=-F\left(x_{1}, x_{2}\right), \quad y=x_{1}$,

where $y=C u$ with $C=\left(\begin{array}{ll}1 & 0\end{array}\right)$. Then the observer has the following form:

$$
\begin{aligned}
\dot{\hat{x}}_{1}= & \hat{x}_{2}+l_{1}\left(x-\hat{x}_{1}\right), \\
\dot{\hat{x}}_{2}= & -d\left(\hat{x}_{1}^{2}-1\right) \hat{x}_{2}-\hat{x}_{1}+l_{2}\left(x-\hat{x}_{1}\right) \\
& +a \cos \omega t+r(t) .
\end{aligned}
$$

The gains $l_{1}$ and $l_{2}$ can be chosen according to the procedure given in $[10,12]$. Now the control input $r$ in (28) can be chosen as (cf. (10) or (13))

$r(t)=-a \cos \omega t+\hat{a} \cos \hat{\omega} t+(d-\hat{d})\left(\hat{x}_{1}^{2}-1\right) \hat{x}_{2}$.

Substituting in (28) we obtain the controlled equation

$\ddot{x}+\hat{d}\left(x^{2}-1\right) \dot{x}+x=\hat{a} \cos \hat{\omega} t+\epsilon(t)$,

where

$\epsilon(t)=(d-\hat{d})\left[\left(\hat{x}_{1}^{2}-1\right) \hat{x}_{2}-\left(x_{1}^{2}-1\right) x_{2}\right]$.

From (3) and (4) it follows that $\epsilon(t)$ decays exponentially to zero. Hence, by choosing the coefficients in (35) appropriately, the dynamical behavior of (28) can be controlled according to the bifurcation structure of (28).

Note that since $x$ is available from measurements, we could use it in (35) as follows:

$r(t)=-a \cos \omega t+\hat{a} \cos \hat{\omega} t+(d-\hat{d})\left(x^{2}-1\right) \hat{x}_{2}$.

Then (36) will remain valid with $\epsilon(t)=(d-\hat{d}) \times$ $\left(x^{2}-1\right)\left(\hat{x}_{2}-x_{2}\right)$.

As a first step in observer design we find the Lipschitz constant. For the parameter values we have used, the original Van der Pol oscillator exhibits different behaviors, such as chaos and period- $n$ limit cycles, while in each case the states of the system are always bounded inside the region $x_{1} \in(-3,3)$ and $x_{2} \in(-10,10)$. Lipschitz constant for this region is found to be $\gamma=180$. The resulting gain vector that satisfies (5) is found to be $L=[2000400000]^{\mathrm{T}}$, entries of which are quite large. Simulating the system with this gain we have seen that the states of the observer converges to the states of the original system quite fast (i.e., in less than $0.1 \mathrm{~s}$ ) as expected. However, before the convergence, the states of the observer undergo an overshooting oscillation whose peak magnitude is also quite large (i.e., 20 times greater than the average magnitude of the states of the original system). For this reason we have chosen a smaller gain vector. To achieve this, we take only the stabilization of the matrix $A_{c}$ into consideration. Choosing the gain vector 


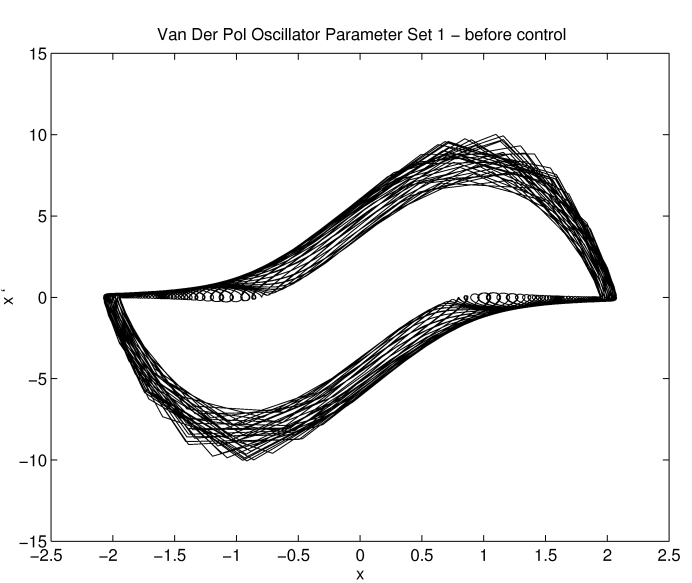

(a)

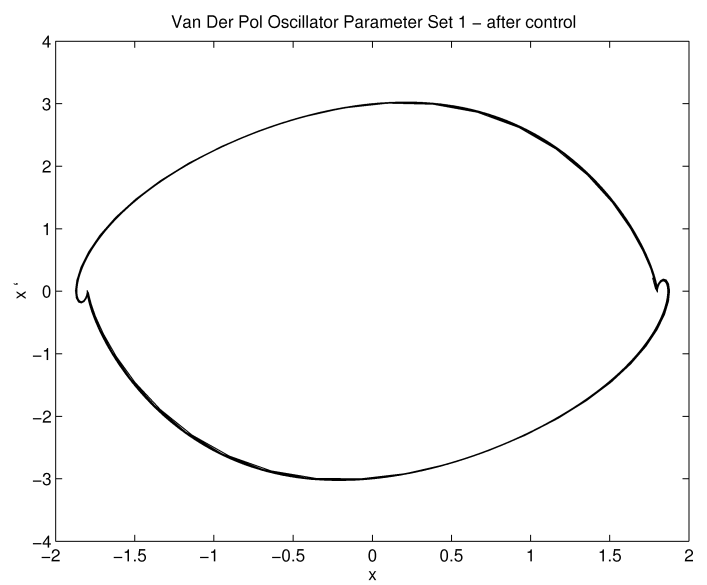

(b)

Fig. 2. State trajectories of the Van der Pol oscillator for set 1: (a) before control, (b) after control.

as $L=\left[\begin{array}{ll}12 & 35\end{array}\right]^{\mathrm{T}}$ is enough to set the eigenvalues of $A_{c}$ at -5 and -7 , and therefore to make $A_{c}$ stable. The observer system shown by Eqs. (33) and (34) works also for these values. From this observation, we have concluded that the procedure given in the previous section and the underlying statements are too conservative. Therefore we use the latter gain vector in our simulations.

We again select two sets of parameter values of the original system from the bifurcation diagrams and former analysis which can be found in [23]. These sets and corresponding system behaviors are as follows:

set $1=(a=2.5, d=6, \omega=3), \quad$ chaos,

set $2=(a=2.5, d=0.5, \omega=3), \quad$ limit cycle.

To switch the chaotic behavior of the system which corresponds to the parameter set 1, shown in Fig. 2(a), we choose the control law as $r(t)=5.5\left(\hat{x}_{1}^{2}-1\right) \hat{x}_{2}$. With this control, the system converges to the limit cycle corresponding the parameter values of set 2 as can be seen from Fig. 2(b).

\subsection{Tracking}

As an example of the tracking problem, let us consider Van der Pol oscillator (28) with the parameter set 1 given above. As a reference trajectory we take $x_{d}(t)=\sin (5 t)$, and assign the control signal using
(21) as

$$
\begin{aligned}
r(t)= & -25 \sin (5 t)-k_{1}\left(\hat{x}_{1}-\sin (5 t)\right) \\
& -k_{2}\left(\hat{x}_{2}+l_{1}\left(x_{1}-\hat{x}_{2}\right)-5 \cos (5 t)\right) \\
& -a \cos (w t)+d\left(\hat{x}_{1}^{2}-1\right) \hat{x}_{2}+\hat{x}_{1} .
\end{aligned}
$$

We simulate the resulting closed loop system using the observer gains $[1235]^{\mathrm{T}}$ as before. Also we choose the coefficients $k_{i}$ in (22) as $k_{1}=15$ and $k_{2}=8$. The resulting behavior is plotted in Fig. 3 .

\section{Conclusions}

In this Letter we presented an observer-based approach to the control of a class of chaotic systems. The class we consider covers the forced oscillators such as Duffing equation and the Van der Pol oscillator. We first transform these systems into a canonical form called the Brunowsky form, hence the method presented in this Letter may also be applied to systems which could be transformed into the Brunowsky form after some modifications. Our aim is to change the dynamics of the given chaotic system appropriately by using the control input to obtain a desirable behavior, i.e., to change from chaos to periodic behavior, or vice versa. To achieve this aim, we use the state observers, which is widely used in the control of dynamical systems. It was shown that under some mild 


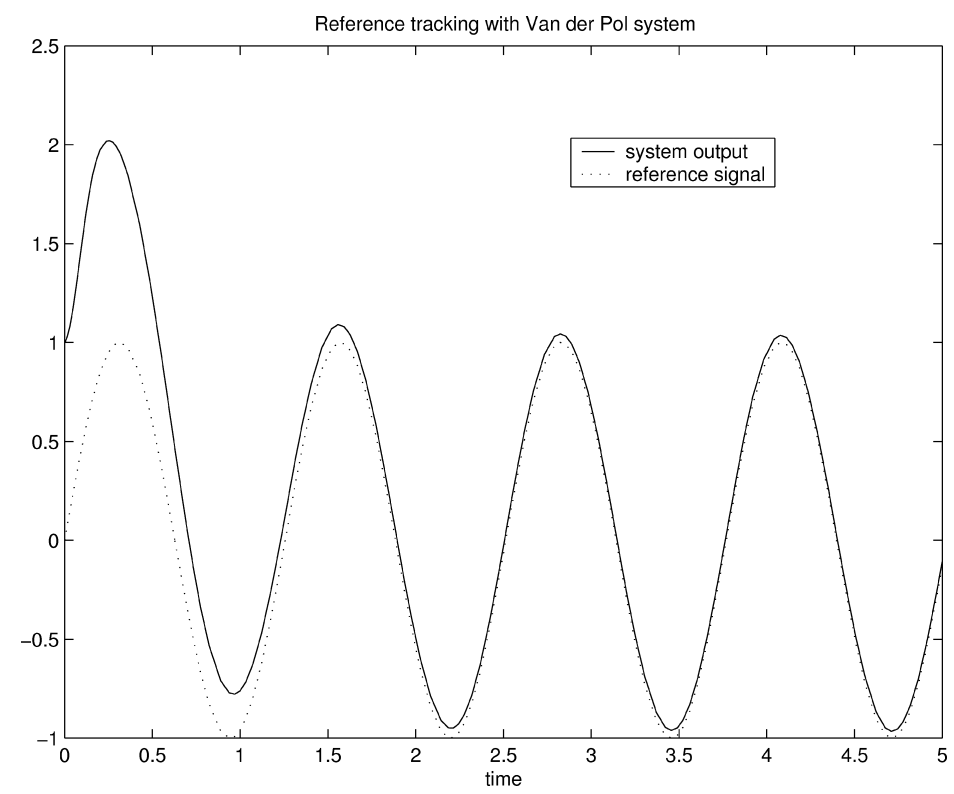

Fig. 3. Output of the Van der Pol oscillator and the reference trajectory $x_{d}(t)=\sin (5 t)$.

conditions exponential convergence of the estimation error to zero is possible and we gave a simple procedure to choose the observer gain. Then the states of the observer were used in a feedback to change the dynamics of the system to be controlled. We also considered the control of the Duffing equation and the Van der Pol oscillator and show that in these cases the parameters which control the dynamical behavior of these systems could be adjusted by the use of an appropriate state observer. We also presented some simulation results.

\section{References}

[1] G. Chen, X. Dong, IEEE Trans. Circuits Syst. 40 (9) (1993).

[2] E. Ott, C. Grebogi, J.A. Yorke, Phys. Rev. Lett. 64 (11) (1990) 1196.

[3] A.Y. Loskutov, A.I. Shismarev, Chaos 4 (2) (1994) 391.

[4] A. Hübler, Helv. Phys. Acta 62 (1989) 343.

[5] U. Dressler, G. Nitsche, Phys. Rev. Lett. 68 (1) (1992) 1.

[6] S. Bhajekar, E. Jonckeere, A. Hammad, $H^{\infty}$ control of chaos, in: 33rd Conference on Decision and Control (Lake Buena Vista), IEEE, 1994, pp. 3285-3286.

[7] P.M. Alsing, A. Gavrielides, V. Kovanis, Phys. Rev. E 49 (2) (1994) 1225.

[8] J.K. John, R.E. Amritkar, Int. J. Bifurcation Chaos 4 (1994) 1687.
[9] G. Chen, Control and synchronization of chaotic systems (a bibliography), 1999, ftp.egr.uh.edu/pub/TeX/chaos.tex, login name: anonymous, password: your e-mail address.

[10] Ö. Morgül, E. Solak, Phys. Rev. E 54 (5) (1996) 4803.

[11] H. Nijmeijer, I.M.Y. Mareels, IEEE Trans. Circuits Syst. 44 (10) (1997) 882.

[12] Ö. Morgül, E. Solak, Int. J. Bifurcation Chaos 7 (6) (1997) 1307.

[13] H. Nijmeijer, H. Berghuis, IEEE Trans. Circuits Syst. 42 (8) (1995) 473.

[14] M. Vidyasagar, Nonlinear Systems Analysis, Prentice-Hall, 1993.

[15] G. Ciccarella, M.D. Mora, A. Germani, Int. J. Control 57 (1993) 537.

[16] A. Tesi, A.D. Angeli, R. Genesio, Int. J. Bifurcation Chaos 4 (6) (1994) 1675.

[17] A.A. Alexeyev, V.D. Shalfeev, Int. J. Bifurcation Chaos 5 (2) (1995) 551.

[18] E. Solak, Ö. Morgül, A gain selection scheme for high-gain nonlinear observers, in: NOLCOS '98, University of Twente, Enschede, The Netherlands, 1998, pp. 757-760.

[19] R. Genesio, A. Tesi, Automatica 28 (3) (1992) 531.

[20] M.W. Hirsch, S. Smale, Differential Equations, Dynamical Systems and Linear Algebra, Academic Press, 1974.

[21] V. Chadran, S. Elgar, C. Pezeshki, Int. J. Bifurcation Chaos 3 (3) (1993) 551.

[22] J.M. Thompson, Nonlinear Dynamics and Chaos, Wiley, 1986.

[23] R. Mettin, U. Parlitz, W. Lauterborn, Int. J. Bifurcation Chaos 3 (1993) 1529. 